Published in final edited form as:

Neurol Clin. 2018 May ; 36(2): 379-394. doi:10.1016/j.ncl.2018.01.008.

\title{
Lambert-Eaton Myasthenic Syndrome
}

\author{
Vita G. Kesner, MD, PhDa, ${ }^{\star}$, Shin J. Oh, MD ${ }^{b}$, Mazen M. Dimachkie, MD ${ }^{c}$, Richard J. Barohn, \\ $M D^{c}$ \\ aNeurology Department, 12 Executive Park Drive NE, Atlanta, GA 30329, USA \\ bUniversity of Alabama at Birmingham, Department of Neurology, SC 350, 1720 2nd Ave South, \\ Birmingham, AL 35294, USA \\ 'Department of Neurology, University of Kansas Medical Center, 3901 Rainbow Boulevard, Mail \\ Stop 2012, Kansas City, KS 66160, USA
}

\section{Keywords}

Lambert-Eaton myasthenic syndrome; Neuromuscular transmission disorder; Paraneoplastic syndrome; P/Q-type voltage-gated calcium channels; 3,4-Diaminopyridine

\section{INTRODUCTION}

The Lambert-Eaton myasthenic syndrome (LEMS) is a paraneoplastic or primary autoimmune neuromuscular junction disorder characterized by proximal weakness and autonomic dysfunction. The characteristic weakness is thought to be caused by antibodies generated against the P/Q-type voltage-gated calcium channels (VGCC) present on presynaptic nerve terminals and by diminished release of acetylcholine (ACh). More than half of LEMS cases are associated with small cell lung carcinoma (SCLC), which expresses functional VGCC. Diagnosis is confirmed by serologic testing and electrophysiologic studies.

\section{HISTORICAL NOTE}

In 1953, Anderson and colleagues ${ }^{1}$ described a 47 -year-old man with fatigable proximal weakness, dysphasia, and diminished Deep Tendon Reflex (DTRs) who improved after an oat cell (small cell) lung cancer was surgically removed. At a meeting of the American Physiologic Society in 1956, Lambert and coworkers ${ }^{2}$ presented a report on six patients with defective neuromuscular transmission associated with malignant neoplasms. They identified some of the clinical and electrophysiologic features that were different from what were expected in typical myasthenia gravis (MG). Subsequently, in 1957, Eaton and Lambert ${ }^{3}$ reported the distinctive electrophysiologic abnormalities seen with repetitive nerve stimulation (RNS) and identified a syndrome that has become known as LEMS. The diagnosis is still based on these electrophysiologic criteria. Soon after the original report, two publications ${ }^{4,5}$ from the same department highlighted the range of clinical and

\footnotetext{
“Corresponding author. vkesner@emory.edu.
} 
electrophysiologic features of this syndrome in 30 patients, including patients without lung cancer. The calcium channels as a target of the pathogenic antibodies in LEMS were first suggested by Fukunaga and colleagues ${ }^{6}$ in 1983 . The discovery of pathogenic autoantibodies to VGCC has facilitated diagnosis and improved the understanding of the pathophysiologic mechanisms leading to LEMS (1983-1995). Subsequent studies showed antibodies against P/Q-type calcium channel as the most prominent in these patients. ${ }^{7}$ Over the past decade, knowledge of epidemiologic and clinical features of LEMS has expanded.

\section{EPIDEMIOLOGY}

LEMS is a rare disorder with annual incidence of only one-tenth to one-fourteenth of that of MG with a prevalence that is 46 times less than that of MG. ${ }^{8}$ Sanders ${ }^{9}$ estimated the prevalence of LEMS in the United States to be 1 in 100,000 on the basis of prevalence of small cell lung cancer. In the paraneoplastic form of the disease (SCLC-LEMS) the median age at onset is 60 years and $65 \%$ to $75 \%$ of patients are men. ${ }^{10}$ The age and sex distribution in nontumor LEMS (NT-LEMS) is similar to that reported for MG. NT-LEMS is seen at all ages, with a peak age of onset of around 35 years and a second, larger peak at age 60 years. Most patients with NT-LEMS are female. ${ }^{10,11}$ The genetic association with HLA-B8-DR3 haplotype is linked to autoimmunity and is present in around $65 \%$ of patients with young onset of NT-LEMS. ${ }^{12}$

\section{TUMOR ASSOCIATION AND IMMUNOPATHOPHYSIOLOGY}

Tumor association is reported in about $60 \%$ of patients with LEMS. ${ }^{11}$ Most patients have SCLC, a smoking-related lung carcinoma with neuroendocrine characteristics, but other malignancies have been described (non-small cell and mixed lung carcinomas, prostate carcinoma, thymoma, and lymphoproliferative disorders). ${ }^{11,13,14}$ The diagnosis of LEMS usually precedes the diagnosis of cancer by many months and even up to 2 years. In a large study of 227 patients, the diagnosis of cancer ranged from 5 years before to 6 years after onset of LEMS. ${ }^{11}$ Weight loss, being male, and a history of smoking are considered to be risk factors. Patients with SCLC associated with LEMS live longer than patients with similar lung cancer stage without a paraneoplastic disorder. An effective immune response directed against cancer is thought to be responsible for this phenomenon. ${ }^{13}$

The initial humoral autoimmune response in patients with LEMS is assumed to be generated against the VGCC subunit antigens on the lung carcinoma. ${ }^{7}, 15$ SCLC expresses VGCC of the N, L, or P type. ${ }^{16}$ VGCC of the P/Q (VGCC-P/Q) and possibly N (VGCC-N) types are targets of IgG-mediated nerve terminal autoimmunity in LEMS. ${ }^{17} \mathrm{P} / \mathrm{Q}$-type VGCCs are involved in ACh release from adult mammalian motor nerve terminals and $\mathrm{N}$-type is responsible for transmitter release from peripheral autonomic nerve terminals. ${ }^{18,19}$

The patient's IgG injected into mice transmits the microelectrophysiologic and ultrastructural changes associated with the disease. Structural alteration of calcium-channelenriched presynaptic membranes and disruption by LEMS-patient serum of depolarizationdependent $\mathrm{Ca}$ influx in cultured small cell carcinoma cells and murine motor nerve terminals were noted on electron microscopy. ${ }^{6,20}$ VGCC antibodies block the ability of calcium to 
flow into the nerve terminal when depolarization occurs, which causes a disruption in the release of ACh. This, in turn, leads to a reduced number of contracting muscles fibers and subsequent weakness.

\section{CLINICAL FEATURES}

The clinical triad typically consists of proximal muscle weakness, autonomic features, and areflexia. ${ }^{14}$ The onset of symptoms is usually gradual and insidious but occasionally it is subacute. Presenting symptoms are leg weakness (60\%), generalized weakness (18\%), muscle pain or stiffness (5\%), dry mouth (5\%), arm weakness (4\%), diplopia (4\%), and dysarthria (2\%). ${ }^{11}$ Others have described muscle weakness in $96 \%$ of 227 patients with LEMS, mostly of the legs, oculobulbar symptoms in 51\%, autonomic symptoms in $49 \%$, respiratory symptoms in $16 \%$, and sensory symptoms in $15 \% .{ }^{11}$

Spreading of weakness in patients with NT-LEMS and SCLC-LEMS was described by Titulaer and coworkers. ${ }^{21}$ Weakness normally spreads proximally to distally, involving feet and hands, and caudally to cranially, finally reaching the oculobulbar region (Fig. 1). This is in contrast to MG, when weakness typically starts cranially and then descends. The speed of progression is much more pronounced in SCLC-LEMS than in NT-LEMS. At the 3-month mark since the onset of symptoms, patients with SCLC-LEMS had proximal legs and arms weakness (see Fig. 1C) but a large percentage of patients with NT-LEMS had only proximal legs weakness (see Fig. 1A). Occurrence of mild cranial nerve symptoms, including diplopia, ptosis, and dysphagia, ranges from $0 \%$ to $80 \% .{ }^{14,22,23}$ Oculobulbar symptoms and/or signs were present in $78 \%$ of patients with LEMS $(n=23)$ evaluated at the Lahey Clinic. ${ }^{23} \mathrm{~A}$ total of $65 \%$ of their patients had ptosis and/or diplopia. In a study of 23 patients with LEMS, bulbar signs and symptoms, including dysarthria, were observed in 10 patients and dysphagia in eight patients. The frequency of ocular and bulbar symptoms in a cohort of 234 patients was reported to be $49 \%$ and $52 \%$, respectively, within 12 months of onset (particularly in patients with SCLC-LEMS). ${ }^{21}$ A retrospective review of the medical records of all patients diagnosed with LEMS at the Mayo Clinic in Rochester, Minnesota was performed with special attention to ophthalmic symptoms and signs. ${ }^{24} \mathrm{~A}$ total of 176 patients were analyzed with the following findings: ophthalmic symptoms included ptosis in $23 \%$, diplopia in $20.5 \%$, decreased vision in $14 \%$, and dry eye complaints in $7 \%$; ophthalmic signs included ptosis in $26 \%$, abnormal ocular motility in $8.5 \%$, strabismus in $8 \%$, pupillary dysfunction in $7 \%$, and findings consistent with dry eyes in $2 \%$. Overall, ocular and bulbar symptoms appear later in the course of the disease of severely affected patients. Although cases of purely ocular symptoms have been reported, ${ }^{25,26}$ isolated ocular weakness is extremely rare and ophthalmoplegia on examination is extremely unusual in LEMS. Respiratory failure is infrequent in LEMS and it is often related to the use of paralytic agents or intercurrent pulmonary pathology, ${ }^{27}$ but artificial ventilation was reported in up to $11 \%$ of cases. ${ }^{11}$

Autonomic dysfunction is reported in $80 \%$ to $96 \%$ of patients with LEMS. ${ }^{14,22,28,29}$ Severe autonomic dysfunction is found on testing even when symptoms are minimal. Both the sympathetic and parasympathetic systems are affected. ${ }^{30,31}$ 
The most common patient complaint is dry mouth. Other symptoms are erectile dysfunction in men, constipation, orthostatic dysfunction, micturition difficulties, dry eyes, and altered perspiration. $^{21}$

Decreased or absent tendon reflexes are typically seen in patients with LEMS. Postexercise facilitation (a short-term return of tendon reflexes and muscle strength to normal range after muscle contraction) is a characteristic (although not very sensitive) phenomenon, which is present in $40 \%$ of patients. ${ }^{32,33}$ Tendon reflexes should be tested after a period of rest because postexercise facilitation phenomenon can mask the hypoactive tendon reflexes.

There are reports of patients having both LEMS and MG. Fifty-five possible cases of MG Lambert-Eaton overlap syndrome were identified through PubMed. ${ }^{34}$ Thirty-nine cases met the universally accepted diagnostic criteria for MG and LEMS. Analysis of clinical features showed that these patients have common MG and LEMS symptoms: oculobulbar paresis and good response to anticholinesterase for MG and limb weakness and decreased or absent reflexes for LEMS. All patients had the classical LEMS pattern in the RNS test: low compound muscle action potential (CMAP) amplitude and incremental response greater than $60 \%$ with brief exercise or at high rate of stimulation. Eight patients had combined positive AChR antibody or muscle-specific kinase antibody and VGCC antibody tests.

\section{DIAGNOSIS}

The diagnosis of LEMS is made based on typical clinical features. Confirmation of diagnosis is based on detection of specific VGCC antibodies and characteristic electrodiagnostic findings.

\section{Voltage-Gated Calcium Channels and SOX1 Antibodies}

The finding of a high serum titer of antibodies against P/Q-type VGCCs strongly supports the diagnosis of Lambert-Eaton syndrome. P/Q VGCC antibodies are present in $80 \%$ to $90 \%$ of patients with LEMS. ${ }^{7,35} \mathrm{P} / \mathrm{Q}$-type VGCCs antibodies have been reported in up to $100 \%$ of patients with LEMS who have small cell lung cancer and in up to $90 \%$ of patients with LEMS who do not have underlying malignancy. More than $90 \%$ of patients belonging to both groups of LEMS (NT-LEMS and SCLC-LEMS) have antibodies against P/Q-type VGCCs.

Antibodies against N-type VGCCs are found more commonly in LEMS associated with primary lung cancer ${ }^{7}$ and, accordingly, the detection of $\mathrm{N}$-type antibodies may increase the possibility of finding an underlying malignancy.

Hajela and coworkers ${ }^{36}$ reported that multiple components of the presynaptic VGCC complex are prospective targets for antibodies in LEMS. Autoantibodies from patients with LEMS bind directly to multiple VGCC a1 subunits and the b3 subunit.

A total of $10 \%$ to $15 \%$ of patients with LEMS have no detectable P/Q VGCC antibodies. Possible explanations have been entertained because the antibodies have lower concentration, different epitopes of VGCC, or antibodies to other proteins. Nakao and colleagues $^{37}$ studied a cohort $(n=17)$ of seronegative patients with clinically definite 
LEMS. The clinical phenotype in this cohort was similar to that in seropositive patients. Incidence of SCLC was only $12 \%$, compared with $60 \%$ to $70 \%$ in seropositive patients. Electrophysiologic features were similar but less prominent. ${ }^{38}$

VGCC autoimmunity (VGCC-P/Q and VGCC-N types) occurs beyond Lambert-Eaton syndrome and lung cancer. Positive VGCC antibodies were reported in less than 5\% of patients with MG and up to $25 \%$ of patients with lung cancer without LEMS. Zalewski and colleagues ${ }^{17}$ reviewed 236 patients at the Mayo Clinic with VGCC antibodies that were found in evaluation for paraneoplastic neurologic autoimmunity. VGCC autoantibodies were detected in $3.4 \%$ of neurologic patients, $1.7 \%$ of healthy control subjects, and $4 \%$ of neurologically asymptomatic lung cancer control subjects. Twenty-one percent of neurologic patients had more than one neoplasm, historically or detected prospectively (SCLC, breast adenocarcinoma, lymphoma, and suspected tonsillar carcinoma). Autoimmune neurologic diagnosis frequencies, including neuromuscular junction disorder, among patients with medium values $(24 \% ; 0.10-0.99 \mathrm{nmol} / \mathrm{L})$ or low values $(19 \% ; 0.03-0.10 \mathrm{nmol} / \mathrm{L})$ were fewer than among patients with antibody values exceeding $1.00 \mathrm{nmol} / \mathrm{L}(71 \% ; P=0.02$ and 0.004 , respectively).

Sixty-four percent of LEMS patients with SCLC also were found to have antibodies against SOX1, an immunogenic tumor antigen in SCLC. Reports suggest that SOX1 may play a role as an early marker of the future predisposition to LEMS/SCLC. ${ }^{39}$ The presence of SOX1 antibodies has a specificity of $95 \%$ for SCLC-LEMS; however, sensitivity is only $65 \%$. Titulaer and colleagues ${ }^{40}$ have found SOX1 antibodies in fewer than $5 \%$ of patients with MG, in up to $25 \%$ of patients with lung cancer without LEMS, and in some patients with systemic lupus erythematosus or rheumatoid arthritis who do not have LEMS but have high levels of circulating immunoglobulins. Patients with LEMS with no evidence of cancer at the initial work-up and positive SOX1 antibodies should have close follow-up.

\section{Electrodiagnostic Studies}

The classic triad of electrophysiologic findings was first defined by Eaton and Lambert. ${ }^{3}$ This triad includes (1) a low CMAP amplitude at rest, (2) a decremental response at low rates of RNS, and (3) an incremental response at high-rate stimulation or after brief exercise (Fig. 2). This typical pattern, however, has not been observed in all cases at the time of initial evaluation and a more diverse electrophysiologic spectrum has been described. ${ }^{41}$

The CMAP in LEMS obtained by supramaximal nerve stimulation is often reduced because many muscle fibers are blocked as a result of decreased quantal release of the transmitter.

At slow rates of stimulation $(2-3 \mathrm{~Hz})$ a decremental response is seen as additional transmitter depletion occurs. Low-rate RNS shows at least 10\% decrement in $94 \%$ to $98 \%$ of patients with LEMS. ${ }^{32,42}$ It has been reported that the decremental pattern with low-rate RNS becomes progressively greater with repeated trains of stimuli in LEMS, whereas in MG, the decrement becomes less pronounced. ${ }^{43}$

After a brief period of voluntary exercise (10-30 seconds), the CMAP in LEMS increases to almost normal size (usually more than double what it was at rest) as a result of calcium 
facilitation of available transmitter release. There is significantly higher diagnostic sensitivity with the 10 -second exercise compared with 30 -second exercise at $100 \%$ increment and $60 \%$ increment levels. ${ }^{44}$ Ten seconds of voluntary isometric contraction has a sensitivity of $84 \%$ to $96 \%$ and is $100 \%$ specific for LEMS. ${ }^{32,44}$ High-rate stimulation (20-50 $\mathrm{Hz}$ ) has comparable sensitivity but is painful. ${ }^{45}$

High-rate RNS or 10 seconds of voluntary isometric contraction shows the CMAP amplitude immediately increases by greater than $100 \%$ in $70 \%$ of patients, consistent with a postactivation facilitation.

A cutoff of $60 \%$ to consider the CMAP increment significant has been proposed, because it raises sensitivity to $97 \%$, whereas specificity remains $99 \%$ in excluding MG. ${ }^{32}$ The authors subsequently found that patients with LEMS with positive VGCC antibodies had lower preexercise amplitudes and higher postexercise facilitation (meeting a $100 \%$ increment as the diagnostic postexercise criterion), whereas all seronegative cases only met the $60 \%$ increment as the diagnostic criterion. ${ }^{38}$ In clinical practice, the finding of 100\% CMAP amplitude increment after brief exercise provides compelling support for the diagnostic confirmation of LEMS.

Despite predominantly proximal limb weakness seen clinically in patients with LEMS, the characteristic RNS abnormalities are seen in distal and in proximal muscle. ${ }^{46}$ In fact, the most sensitive muscles for detecting characteristic electrophysiologic abnormalities of low resting CMAP amplitude and increment of more than $100 \%$ after 10 seconds maximal voluntary contraction are abductor digiti minimi, abductor pollicis brevis, and anconeus. ${ }^{47}$ Thus, it is usually preferable and adequate enough to do the RNS test on distal muscles, either on the abductor pollicis brevis or abductor digiti minimi muscle.

RNS can be abnormal in other neuromuscular disorders and conventional needle electromyography (EMG) should be considered to exclude a muscle and anterior horn cell diseases. Needle EMG in LEMS demonstrates unstable motor unit action potential.

Single fiber EMG (SFEMG) findings are similar to postsynaptic neuromuscular junction disorders with increased jitter and blocking; however, these findings are frequently out of proportion to the severity of weakness. ${ }^{48}$ The relationship between electrodiagnostic findings and clinical severity was published by $\mathrm{Oh}$ and colleagues ${ }^{49}$ who analyzed 82 SFEMG tests in the extensor digitorum communis muscle in 30 patients with LEMS. Jitter was abnormal in all patients at the first evaluation regardless of clinical severity and it was increasingly abnormal with worsening disease severity. Mean consecutive difference correlated well with clinical and electrophysiologic severity on RNS test. Stimulated SFEMG was performed in the extensor digitorum communis muscle and in LEMS and LEMS/MG overlap, jitter and blocking improve with stimulation rates, as compared with opposite effect in MG. ${ }^{50}$

\section{SCREENING FOR SMALL CELL LUNG CANCER CARCINOMA}

When the diagnosis of LEMS has been confirmed, an extensive search for malignancy should be carried out. A computed tomography scan or MRI of the chest or a PET scan is 
recommended for initial evaluation of patients. Screening for cancer should continue every 3 to 6 months for at least 2 years. According to Titulaer and colleagues, ${ }^{51}$ screening detected 91\% of SCLC within 3 months and 96\% within 1 year of diagnosis of LEMS. Clinical Dutch-English LEMS Tumor Association prediction score (DELTA-P) was published in $2011 .{ }^{10}$ A DELTA-P score was derived allocating one point for the presence of each of the following items at or within 3 months from onset: age at onset greater than or equal to 50 years, smoking at diagnosis, weight loss greater than or equal to $5 \%$, bulbar involvement, erectile dysfunction, and Karnofsky performance status lower than 70 . The probability for SCLC is calculated at diagnosis of LEMS, and varies from $0.2 \%$ to $6 \%$ with a DELTA-P score of 0 to 1 , up to $83.9 \%$ to $100 \%$ with a score of 3 to 6 . Titulaer and colleagues proposed a screening strategy with computed tomography thorax and F-fluorodeoxy-glucose-PET based on a DELTA-P score. In patients with a DELTA-P score of 0 or 1 , it was suggested that screening be discontinued after two adequate and negative screens 6 months apart (the chance of SCLC is lower than 1 per 1000). If the score is 2 , screening should continue every 6 months for 2 years. If the score is 3 to 6 , the second screening should be performed after 3 months and it should be repeated every 6 months afterward for 2 years (Fig. 3).

\section{TREATMENT}

Treatment of patients with LEMS includes treatment of a tumor when applicable and symptomatic management. AChE inhibitors do not usually produce significant improvement in LEMS, although they may improve dry mouth. Lambert, in his first report, noted the poor response of his patients to neostigmine. ${ }^{2}$ For symptomatic treatment, pyridostigmine, guanidine, 4-aminopyridine, and 3,4-diaminopyridine (3,4-DAP) have been tried. ${ }^{52,53}$ Except for 3,4-DAP, these compounds have been studied in small open label case series, but not in clinical trials.

3,4-DAP blocks VGCCs that lead to prolongation of depolarization of the action potential at motor nerve terminals and increase the open time of the VGCCs. ${ }^{54}$ This process results in increased presynaptic influx of calcium and enhancement of ACh release manifesting by improvement in muscle function. Typical dosage of prescribed 3,4-DAP is $10 \mathrm{mg}$ by mouth three times a day. Maximum recommended dosage is $80 \mathrm{mg}$ a day.

McEvoy and colleagues ${ }^{55}$ in 1989 studied oral 3,4-DAP in a double-blind, randomized, crossover controlled trial in 12 patients with LEMS, seven of whom had cancer. Results of this trial showed significant improvement in neurologic disability score, isometric myometry limb testing strength measures, CMAP amplitude change, and autonomic function change following oral administration of up to $100 \mathrm{mg}$ per day 3,4-DAP compared with the placebo. One patient had a seizure after 10 months of treatment. Another randomized, double-blind, crossover trial of oral 3,4-DAP of up to $80 \mathrm{mg}$ per day showed significant efficacy over the placebo in seven patients with LEMS. ${ }^{56}$ Results of the parallel group trial of 26 cases by Sanders and colleagues ${ }^{57}$ showed similar results, namely significant improvement of the Quantitative Myasthenia Gravis (QMG) score and resting amplitude following the oral administration of $60 \mathrm{mg}$ per day 3,4-DAP. 
Intravenous administration of 3,4-DAP produced similar results. Wirtz and colleagues ${ }^{58}$ reported no additional benefit with the addition of pyridostigmine, and pyridostigmine in isolation showed no difference to the placebo group.

The 2011 Cochrane review ${ }^{59}$ described the results of four of the previously described randomized controlled trials in a total of 54 patients with LEMS. This review concluded that there was "limited but moderate to high quality evidence showing that over days 3,4-DAP improved muscle strength and CMAP in LEMS." All trials reported a significant improvement in muscle strength score, QMG score, or CMAP amplitude after treatment. Also, 3,4-DAP was well tolerated. The most common side effects are perioral tingling, digital paresthesias, and gastrointestinal symptoms. The most serious adverse events are dose-dependent. Seizures were described at doses of more than $100 \mathrm{mg}$ per day and supraventricular tachycardia with iatrogenic intoxication of $360 \mathrm{mg}$. Prolongation of the QT interval is often mentioned as a possible side effect.

3,4-DAP was first approved for use in Europe for patients with LEMS in December 2009. It has been recommended as a first-line symptomatic treatment of LEMS by the European Federation of Neurologic Societies since 2010. An algorithm for treatment of LEMS was proposed by Titulaer and coworkers (Fig. 4). ${ }^{21}$

Of the two recently completed studies of 3,4-DAP in LEMS, one has been published so far. At present, 3,4-DAP base has not received Food and Drug Administration approval for the symptomatic management of LEMS in the United States. It has limited availability through Treatment Investigational New Drugs studies, expanded-access programs, clinical trials, and compounding pharmacies. Amifampridine phosphate (the salt form of 3,4-DAP) has been shown to have superior stability (it can be stored at room temperature) compared with the base. ${ }^{60} \mathrm{An}$ oral formulation containing amifampridine phosphate, equivalent to $10 \mathrm{mg}$ base, has been developed (Firdapse, Catalyst Pharmaceuticals, Coral Gables, FL). Amifampridine phosphate has received orphan drug designation and has been granted a Breakthrough Therapy designation by the Food and Drug Administration in 2013.

A phase 3 clinical trial evaluated the efficacy and safety of amifampridine phosphate for symptomatic treatment of LEMS. ${ }^{61}$ A multicenter, randomized phase 3 "withdrawal trial" was conducted at 18 sites in the United States, the European Union, and the Russian Federation. Total daily dose of amifampridine phosphate was 15 to $80 \mathrm{mg} / \mathrm{d}$, given in 3 to 4 divided doses, with a maximum single dose of $20 \mathrm{mg}$. Thirty-eight patients were randomized and the efficacy of amifapridine phosphate at Day 8 and Day 14 was evaluated. The coprimary efficacy end points were the change from baseline to Day 14 in Quantitative Myasthenia Gravis score and in the Subject Global Impression of change. Secondary end points included the changes from baseline in Clinical Global Impression of Improvement and Timed 25-foot-walk-test speed at Day 14. Tertiary end point was the CMAP in the abductor digiti minimi muscle at rest with a single maximal stimulation of the ulnar nerve at the wrist. The primary efficacy end points and one (Clinical Global Impression of Improvement) of the secondary efficacy end points were met, showing a significant benefit of amifampridine phosphate over the placebo at Day 14. Amifampridine phosphate was well tolerated. The most common adverse events were oral (40\%) and digital (34\%) paresthesias, 
nausea, and headache. One limitation of this trial is that the protocol was not followed by 10 patients. This study provided class I evidence of efficacy of amifampridine phosphate as a symptomatic treatment of LEMS.

For rapidly progressive LEMS symptoms, intravenous immunoglobulin (IVIG) has been used. A single randomized double-blind, placebo-controlled, crossover study involving patients with LEMS with no carcinoma showed a significant improvement in limb strength (measured by myometry) peaking at 2 to 4 weeks and effects lasting up to 8 weeks following IVIG treatment ( $1 \mathrm{~g} / \mathrm{kg}$ body weight/day for 2 days). The clinical response was associated with a significant decline in antibody titer to VGCC. ${ }^{62}$ Aside from this trial, there are few data regarding the use of IVIG treatment of LEMS. Experience from case reports indicates that IVIG is useful in patients with LEMS, as a short- and long-term repeated treatment, especially when immunosuppressive drugs are not fully effective. ${ }^{63-65}$

For patients with severe symptoms requiring a prompt management, plasma exchange delivered as a 5-day course, removing 3 to $4 \mathrm{~L}$ each day, typically improves LEMS symptoms in cancer and noncancer LEMS. Objective CMAP amplitude outcome measures corresponded to clinical improvement. The peak beneficial effect is usually demonstrated after about 2 weeks, subsiding after 6 weeks. ${ }^{66}$

In patients whose LEMS symptoms are not adequately controlled on symptomatic treatment, long-term oral immunosuppression with prednisolone and azathioprine is usually considered, ${ }^{67}$ with treatment regimens based on previous trial data in MG (prednisolone dose is up to $1.5 \mathrm{mg} / \mathrm{kg}$ on alternate days; azathioprine a total dose of $2.5 \mathrm{mg} / \mathrm{kg}$ per day) showing beneficial effects from combined therapy. The effectiveness of the combined prednisone-azathioprine therapy has only been shown in a retrospective study and part prospective study. ${ }^{68}$ Observational follow-up data on 47 patients with NT-LEMS in one center demonstrated that combination therapy of prednisolone and azathioprine was required in proximately $90 \%$ patients, with $43 \%$ achieving sustained clinical remission within the first 3 years of treatment. However, prednisolone (mean, $30 \mathrm{mg}$ on alternate days, often combined with azathioprine) was still required after 3-year follow-up, with only $14 \%$ achieving clinical and pharmacologic remission.

Similar data with modest benefit of prednisone and azathioprine were published from other centers. ${ }^{69}$ Observational studies on 73 patients with LEMS (42\% with lung cancer) have previously demonstrated that high-dose prednisolone often achieved only mild to moderate improvement in symptoms, which was not sustained when the dose was reduced. ${ }^{52}$

There are isolated reports of medium-term benefit from rituximab ${ }^{70}$ and favorable response to rituximab. ${ }^{71,72}$ There is a theoretic concern that immunosuppression may reduce the immunologic suppression of tumor growth. Aggressive immunotherapy is more commonly prescribed in patients without cancer. The specific treatment of the underlying tumor usually ultimately results in improvement or even remission of symptoms but symptomatic treatment early on is extremely important for patients to regain and maintain strength and function. 
Unlike other paraneoplastic syndromes, which are usually resistant to any therapy, LEMS is known to be consistently responsive to immunotherapy or anticancer therapy. ${ }^{73}$ Thus, in patients with the paraneoplastic form of LEMS, it is critical that antitumor treatments are given to treat underlying malignancies. Clinical data suggest that the immune response associated with LEMS may suppress tumor activity, thus prolonging survival times of LEMS patients with cancer. ${ }^{13,74}$ This was confirmed in a recent prospective study of 31 LEMS patients with SCLC who were compared with 279 SCLC without neurologic illness. After controlling for known SCLC prognostic factors, the presence of LEMS with SCLC conferred a significant survival advantage, thereby excluding lead time bias as a potential alternate explanation. ${ }^{75}$ Chemotherapeutic agents, such as vincristine, doxorubicin, and cyclophosphamide, are effective against SCLC-associated LEMS, but the proportions of patients benefiting are small. In a study of 16 patients with LEMS associated with small cell carcinoma, 13 patients received specific tumor therapy and most also received pharmacologic and immunologic treatment of LEMS. ${ }^{73}$

Seven of 11 patients surviving for more than 2 months after tumor therapy showed substantial neurologic improvement, but only one patient was in complete remission 7 years after the cancer therapy. In 3 of these 11 patients, improvement was only transient.

\section{SUMMARY}

LEMS is a rare and unique autoimmune calcium channelopathy. An underlying small cancer is found in more than half of patients with LEMS. Continued cancer screening is required every 3 to 6 months for at least 2 years following symptom onset. Proximal muscle weakness, autonomic features, and areflexia are a typical clinical triad. Characteristic electrophysiologic findings include reduced CMAP amplitudes; decremental response at low-rate RNS; and most importantly an incremental response immediately following 10 seconds of maximal voluntary contraction or in severely weak cases, brief $50 \mathrm{~Hz}$ RNS. 3,4DAP dosed at 30 to $80 \mathrm{mg}$ daily is the most effective symptomatic treatment of LEMS with good tolerability and safety profile.

\section{Acknowledgments}

Disclosures: Dr. M.M. Dimachkie is on the speaker's bureau or is a consultant for Alnylam, Baxalta, Catalyst, CSLBehring, Mallinckrodt, Novartis, NuFactor, and Terumo. He has also received grants from Alexion, Biomarin, Catalyst, CSL Behring, FDA/OPD, GSK, Grifols, MDA, NIH, Novartis, Orphazyme, Sanofi, and TMA. Dr R.J. Barohn is a consultant for NuFactor and is on the advisory board for Novartis. He has received an honorarium from Option Care and PlatformQ Health Education. He has received research grants from NIH, FDA/OOPD, NINDS, Novartis, Sanofi/Genzyme, Biomarin, IONIS, Teva, Cytokinetics, Eli Lilly, PCORI, ALSA, and PTC. This work was supported by a CTSA grant from NCATS awarded to the University of Kansas for Frontiers: University of Kansas Clinical and Translational Science Institute (\# UL1TR002366) The contents are solely the responsibility of the authors and do not necessarily represent the official views of the NIH or NCATS.

\section{REFERENCES}

1. Anderson HJ, Churchill-Davidson HC, Richardson AT. Bronchial neoplasm with myasthenia: prolonged apnea after administration of succinylcholine. Lancet 1953;265:1291-3. [PubMed: 13110148]

2. Lambert EH, Eaton LM, Rooke ED. Defect of neuromuscular conduction associated with malignant neoplasms. Am J Physiol 1956;187:612-3. 
3. Eaton LM, Lambert EH. Electromyography and electrical stimulation of nerves in diseases of the motor unit: observations on a myasthenic syndrome associated with malignant tumours. J Am Med Assoc 1957;163:1117-24. [PubMed: 13405774]

4. Rooke ED, Eaton LM, Lambert EH, et al. Myasthenia and malignant intrathoracic tumor. Med Clin North Am 1960;44:977-88. [PubMed: 14438718]

5. Lambert EH, Rooke ED. Myasthenic state and lung cancer In: Brain WR, Norris FH Jr, editors. The remote effect of cancer on the nervous system. New York: Grune\&Stratton; 1965 p. 67-80.

6. Fukunaga H, Engel AG, Lang B, et al. Paucity and disorganization of presynaptic membrane active zones in the Lambert-Eaton myasthenic syndrome. Muscle Nerve 1982;5:686-97.

7. Lennon VA, Kryzer TJ, Griesmann GE, et al. Calcium-channel antibodies in the Lambert-Eaton syndrome and other paraneoplastic syndromes. N Engl J Med 1995;332:1467-74. [PubMed: 7739683]

8. Wirtz PW, Nijnuis MG, Sotodeh M, et al. Dutch Myasthenia Study Group. The epidemiology of myasthenia gravis, Lambert-Eaton myasthenic syndrome and their associated tumours in the northern part of the province of South Holland. J Neurol 2003;250(6):698-701. [PubMed: 12796832]

9. Sanders DB. Lambert-Eaton myasthenic syndrome: diagnosis and treatment. Ann N Y Acad Sci 2003;998:500-8. [PubMed: 14592920]

10. Titulaer MJ, Maddison P, Sont JK, et al. Clinical Dutch-English Lambert-Eaton myasthenic syndrome (LEMS) tumor association prediction score accurately predicts small-cell lung cancer in the LEMS. J Clin Oncol 2011;29:902-8. [PubMed: 21245427]

11. Wirtz PW, Smallegange TM, Wintzen AR, et al. Differences in clinical features between the Lambert-Eaton myasthenic syndrome with and without cancer: an analysis of 227 published cases. Clin Neurol Neurosurg 2002;104:359-63. [PubMed: 12140105]

12. Wirtz PW, Willcox N, van der Slik AR, et al. HLA and smoking in prediction and prognosis of small cell lung cancer in autoimmune Lambert-Eaton myasthenic syndrome. J Neuroimmunol 2005;159:230-7. [PubMed: 15652424]

13. Titulaer MJ, Verschuuren JJ. Lambert-Eaton myasthenic syndrome: tumor versus nontumor forms. Ann NY Acad Sci 2008;1132:129-34. [PubMed: 18567862]

14. O'Neill JH, Murray NMF, Newsom-Davis J. The Lambert-Eaton myasthenic syndrome: a review of 50 cases. Brain 1988;111:577-96. [PubMed: 2838124]

15. Meriney SD, Hulsizer SC, Lennon VA, et al. Lambert-Eaton myasthenic syndrome immunoglobulins react with multiple types of calcium channels in small-cell lung carcinoma. Ann Neurol 1996;40:739-49. [PubMed: 8957015]

16. Oguro-Okano M, Griesmann GE, Wieben ED, et al. Molecular diversity of neuronal-type calcium channels identified in small cell lung carcinoma. Mayo Clin Proc 1992;67:1150-9. [PubMed: 1335101]

17. Zalewski NL, Lennon VA, Lachance DH, et al. P/Q- and N-type calcium-channel antibodies: oncological, neurological, and serological accompaniments. Muscle Nerve 2016;54(2):220-7. [PubMed: 26789908]

18. Katz E, Ferro PA, Weiss G, et al. Calcium channels involved in synaptic transmission at the mature and regenerating mouse neuromuscular junction. J Physiol 1996;497:687-9. [PubMed: 9003554]

19. Hirning LD, Fox AP, McCleskey EW, et al. Dominant role of N-type Ca21 channels in evoked release of norepinephrine from sympathetic neurons. Science 1998;239:57-61.

20. Lang B, Newsom-Davis J, Wray D, et al. Autoimmune etiology for myasthenic (Eaton-Lambert) syndrome. Lancet 1981;2:224-6. [PubMed: 6114283]

21. Titulaer MJ, Lang B, Verschuuren JJ. Lambert-Eaton myasthenic syndrome: from clinical characteristics to therapeutic strategies. Lancet Neurol 2011;10(12): 1098-107. [PubMed: 22094130]

22. Titulaer MJ, Wirtz PW, Kuks JB, et al. The Lambert-Eaton myasthenic syndrome 1988-2008: a clinical picture in 97 patients. J Neuroimmunol 2008;201-02:153-8.

23. Burns TM, Russell JA, LaChance DH, et al. Oculobulbar involvement is typical with LambertEaton myasthenic syndrome. Ann Neurol 2003;53:270-3. [PubMed: 12557298] 
24. Young JD, Leavitt JA. Lambert Eaton myasthenic syndrome: ocular signs and symptoms. J Neuroophthalmol 2016;36:20-2. [PubMed: 25993122]

25. Oh SJ. The Eaton-Lambert syndrome in ocular myasthenia gravis. Arch Neurol 1974;31:183-6. [PubMed: 4855164]

26. Rudnicki SA. Lambert-Eaton myasthenic syndrome with pure ocular weakness. Neurology 2007;68:1863-4. [PubMed: 17515551]

27. Smith AG, Wald J. Acute ventilatory failure in Lambert-Eaton myasthenic syndrome and its response to 3,4-diaminopyridine. Neurology 1996;46:1143-5. [PubMed: 8780107]

28. Lorenzoni PJ, Scola RH, Kay CS, et al. Non-paraneoplastic Lambert-Eaton myasthenic syndrome: a brief review of 10 cases. Arq Neuropsiquiatr 2010;68:849-54. [PubMed: 21243240]

29. Pellkofer HL, Armbruster L, Linke R, et al. Managing non-paraneoplastic Lambert-Eaton myasthenic syndrome: clinical characteristics in 25 German patients. J Neuroimmunol 2009;217:90-4. [PubMed: 19833394]

30. Waterman SA, Lang B, Newsom-Davis J. Effect of Lambert-Eaton myasthenic syndrome antibodies on autonomic neurons in the mouse. Ann Neurol 1997;42:147-56. [PubMed: 9266723]

31. Khurana RK, Koski CL, Mayer RF. Autonomic dysfunction in Lambert-Eaton myasthenic syndrome. J Neurol Sci 1988;85:77-86. [PubMed: 2838585]

32. Oh SJ, Kurokawa K, Claussen GC, et al. Electrophysiological diagnostic criteria of Lambert-Eaton myasthenic syndrome. Muscle Nerve 2005;32:515-20. [PubMed: 16003742]

33. Odabasi Z, Demirci M, Kim DS, et al. Postexercise facilitation of reflexes is not common in Lambert-Eaton myasthenic syndrome. Neurology 2002;59:1085-7. [PubMed: 12370470]

34. Oh SJ. Myasthenia gravis Lambert-Eaton overlap syndrome. Muscle Nerve 2016; 53:20-6. [PubMed: 26418033]

35. Motomura M, Lang B, Johnston I, et al. Incidence of serum anti-P/Q-type and anti-N-type calcium channel autoantibodies in the Lambert-Eaton myasthenic syndrome. J Neurol Sci 1997;147:35-42. [PubMed: 9094058]

36. Hajela RK, Huntoon KM, Atchison WD. Lambert-Eaton syndrome antibodies target multiple subunits of voltage-gated Ca2+ channels. Muscle Nerve 2015; 51(2):176-84. [PubMed: 24862203]

37. Nakao YK, Motomura M, Fukudome T, et al. Seronegative Lambert-Eaton myasthenic syndrome. Neurology 2002;59:1773-5. [PubMed: 12473768]

38. Oh SJ, Hatanaka Y, Claussen GC, et al. Electrophysiological differences in seropositive and seronegative Lambert-Eaton myasthenic syndrome. Muscle Nerve 2007;35:178-83. [PubMed: 17058271]

39. Sabater L, Titulaer M, Saiz A, et al. SOX1 antibodies are markers of paraneoplastic Lambert-Eaton myasthenic syndrome. Neurology 2008;70:924-8. [PubMed: 18032743]

40. Titulaer MJ, Klooster R, Potman M, et al. SOX antibodies in small-cell lung cancer and LambertEaton myasthenic syndrome: frequency and relation with survival. J Clin Oncol 2009;27:4260-7. [PubMed: 19667272]

41. Oh SJ. Diverse electrophysiological spectrum of the Lambert-Eaton myasthenic syndrome. Muscle Nerve 1989;12:464-9. [PubMed: 2725573]

42. Tim RW, Massey JM, Sanders DB. Lambert-Eaton myasthenic syndrome (LEMS): clinical and electrodiagnostic features and response to therapy in 59 patients. Ann N Y Acad Sci 1998;841:823-6. [PubMed: 9668336]

43. Sanders DB, Cao L, Massey JM, et al. Is the decremental pattern in Lambert-Eaton syndrome different from that in myasthenia gravis? Clin Neurophysiol 2014;125(6):1274-7. [PubMed: 24332471]

44. Hatanaka Y, Oh SJ. Ten-second exercise is superior to 30-second exercise for post-exercise facilitation in diagnosing Lambert-Eaton myasthenic syndrome. Muscle Nerve 2008;37:572-5. [PubMed: 18288711]

45. Tim RW, Sanders DB. Repetitive nerve stimulation studies in the Lambert-Eaton myasthenic syndrome. Muscle Nerve 1994;17(9):995-1001. [PubMed: 8065402]

46. Oh SJ. Electromyography: neuromuscular transmission studies. Baltimore (MD): Williams \& Wilkins; 1988. 
47. Maddison P, Newsom-Davis J, Mills KR. Distribution of electrophysiological abnormality in Lambert-Eaton myasthenic syndrome. J Neurol Neurosurg Psychiatry 1998;65:213-7. [PubMed: 9703174]

48. Sanders DB, Stålberg EV. AAEM minimonograph \#25: single-fiber electromyography. Muscle Nerve 1996;19:1069-83. [PubMed: 8761262]

49. Oh SJ, Ohira M. Single-fiber EMG and clinical correlation in Lambert-Eaton myasthenic syndrome. Muscle Nerve 2013;47(5):664-7. [PubMed: 23505075]

50. Chaudhry V, Watson DF, Bird SJ, et al. Stimulated single-fiber electromyography in LambertEaton myasthenic syndrome. Muscle Nerve 1991;14(12):1227-30. [PubMed: 1662773]

51. Titulaer MJ, Wirtz PW, Willems LN, et al. Screening for small-cell lung cancer: a follow-up study of patients with Lambert-Eaton myasthenic syndrome. J Clin Oncol 2008;26:4276-81. [PubMed: 18779614]

52. Tim RW, Massey JM, Sanders DB. Lambert-Eaton myasthenic syndrome: electrodiagnostic finding and response to treatment. Neurology 2000;54:2176-8. [PubMed: 10851390]

53. Oh SJ, Kim DS, Head TC, et al. Low-dose guanidine and pyridostigmine: relatively safe and effective long-term symptomatic therapy in Lambert-Eaton myasthenic syndrome. Muscle Nerve 1997;20:1146-52. [PubMed: 9270671]

54. Molgo J, Lundh H, Thesle S. Potency of 3,4-diaminopyridine and 4-aminopyridine on mammalian neuromuscular transmission and the effect of $\mathrm{pH}$ changes. Eur J Pharmacol 1980;61:25-34. [PubMed: 6101553]

55. McEvoy KM, Windebank AJ, Daube JR, et al. 3,4-Diaminopyridine in the treatment of LambertEaton myasthenic syndrome. N Engl J Med 1989;321:1567-71. [PubMed: 2555713]

56. Oh SJ, Claussen GG, Hatanaka Y, et al. 3,4-Diaminopyridine is more effective than placebo in a randomized, double-blind, cross-over drug study in LEMS. Muscle Nerve 2009;40:795-800. [PubMed: 19722254]

57. Sanders DB, Massey JM, Sanders LL, et al. A randomized trial of 3,4-diaminopyridine in LambertEaton myasthenic syndrome. Neurology 2000;54(3):603-7. [PubMed: 10680790]

58. Wirtz PW, Verschuuren JJ, van Dijk JG, et al. Efficacy of 3,4-diaminopyridine and pyridostigmine in the treatment of Lambert-Eaton myasthenic syndrome: a randomized, double-blind, placebocontrolled, crossover study. Clin Pharmacol Ther 2009;86(1):44-8. [PubMed: 19357643]

59. Keogh M, Sedehizadeh S, Maddison P. Treatment for Lambert-Eaton myasthenic syndrome. Cochrane Database Syst Rev 2011;(2):CD003279. [PubMed: 21328260]

60. Raust JA, Goulay-Dufay S, Le Hoang MD, et al. Stability studies of ionised and non-ionised 3,4diaminopyridine: hypothesis of degradation pathways and chemical structure of degradation products. J Pharm Biomed Anal 2007;43:83-8. [PubMed: 16844337]

61. Oh SJ, Shcherbakova N, Kostera-Pruszczyk A, et al. Amifampridine phosphate (Firdapse(®)) is effective and safe in a phase 3 clinical trial in LEMS. Muscle Nerve 2016;53(5):717-25. [PubMed: 26852139]

62. Bain PG, Motomura M, Newsom-Davis J, et al. Effects of intravenous immunoglobulin on muscle weakness and calcium-channel autoantibodies in the Lambert-Eaton myasthenic syndrome. Neurology 1996;47:678-83. [PubMed: 8797464]

63. Illa I IVIg in myasthenia gravis, Lambert Eaton myasthenic syndrome and inflammatory myopathies: current status. J Neurol 2005;252(Suppl 1):I14-8. [PubMed: 15959667]

64. Muchnik S, Losavio AS, Vidal A, et al. Long-term follow-up of Lambert-Eaton syndrome treated with intravenous immunoglobulin. Muscle Nerve 1997;20(6):674-8. [PubMed: 9149073]

65. Bird SJ. Clinical and electrophysiologic improvement in Lambert-Eaton syndrome with intravenous immunoglobulin therapy. Neurology 1992;42:1422-3. [PubMed: 1620360]

66. Newsom-Davis J, Murray NM. Plasma exchange and immunosuppressive drug treatment in the Lambert-Eaton myasthenic syndrome. Neurology 1984;34:480-5. [PubMed: 6322050]

67. Newsom-Davis J A treatment algorithm for Lambert-Eaton myasthenic syndrome. Ann NY Acad Sci 1998;841:817-22. [PubMed: 9668335]

68. Maddison P, Lang B, Mills K, et al. Long term outcome in Lambert-Eaton myasthenic syndrome without lung cancer. J Neurol Neurosurg Psychiatry 2001; 70(2):212-7. [PubMed: 11160470] 
69. Maddison P Treatment in Lambert-Eaton myasthenic syndrome. Ann N Y Acad Sci 2012;1275:7884. [PubMed: 23278581]

70. Maddison P, McConville J, Farrugia ME, et al. The use of rituximab in myasthenia gravis and Lambert-Eaton myasthenic syndrome. J Neurol Neurosurg Psychiatry 2011;82(6):671-3. [PubMed: 20392977]

71. Pellkofer HL, Voltz R, Kuempfel T. Favorable response to rituximab in a patient with anti-VGCCpositive Lambert-Eaton myasthenic syndrome and cerebellar dysfunction. Muscle Nerve 2009;40(2):305-8. [PubMed: 19609921]

72. Boutin E, Rey C, Romeu M, et al. Favourable outcome after treatment with rituximab in a case of seronegative non-paraneoplastic Lambert-Eaton myasthenic syndrome. Rev Med Interne 2013;34(8):493-6. [PubMed: 23759215]

73. Chalk CH, Murray NM, Newsome-Davis J, et al. Responses of the Lambert-Eason myasthenic syndrome to treatment of associated small-cell lung cancer. Neurology 1990;40:1552-6. [PubMed: 2170866]

74. Maddisson P, Newsome-Davis J, Mills KR, et al. Favorable prognosis in Lambert-Eaton myasthenic syndrome and small-cell lung carcinoma. Lancet 1999;353:117-8.

75. Maddison P, Gozzard P, Grainge MJ, et al. Long-term survival in paraneoplastic Lambert-Eaton myasthenic syndrome. Neurology 2017;88(14):1334-9. [PubMed: 28251917] 


\section{KEY POINTS}

- LEMS is a paraneoplastic or primary autoimmune neuromuscular junction disorder. The antigenic target is the P/Q-type voltage-gated calcium channel (VGCC), which plays a role in decreasing the release of acetylcholine.

- $\quad$ The clinical triad typically consists of proximal muscle weakness, autonomic features, and areflexia.

- Characteristic electrophysiologic triads are the low compound muscle action potentials (CMAP), decremental response at the low-rate stimulation, and incremental response with 10 seconds maximal voluntary contraction or highrate stimulation.

- $\quad$ Paraneoplastic form of LEMS occurs almost always with an underlying small cell lung cancer (SCLC). Cancer screening should continue every 3 to 6 months for at least 2 years from symptom onset.

- $\quad$ Effective symptomatic treatment of LEMS is with 3,4-diaminopyridine. 

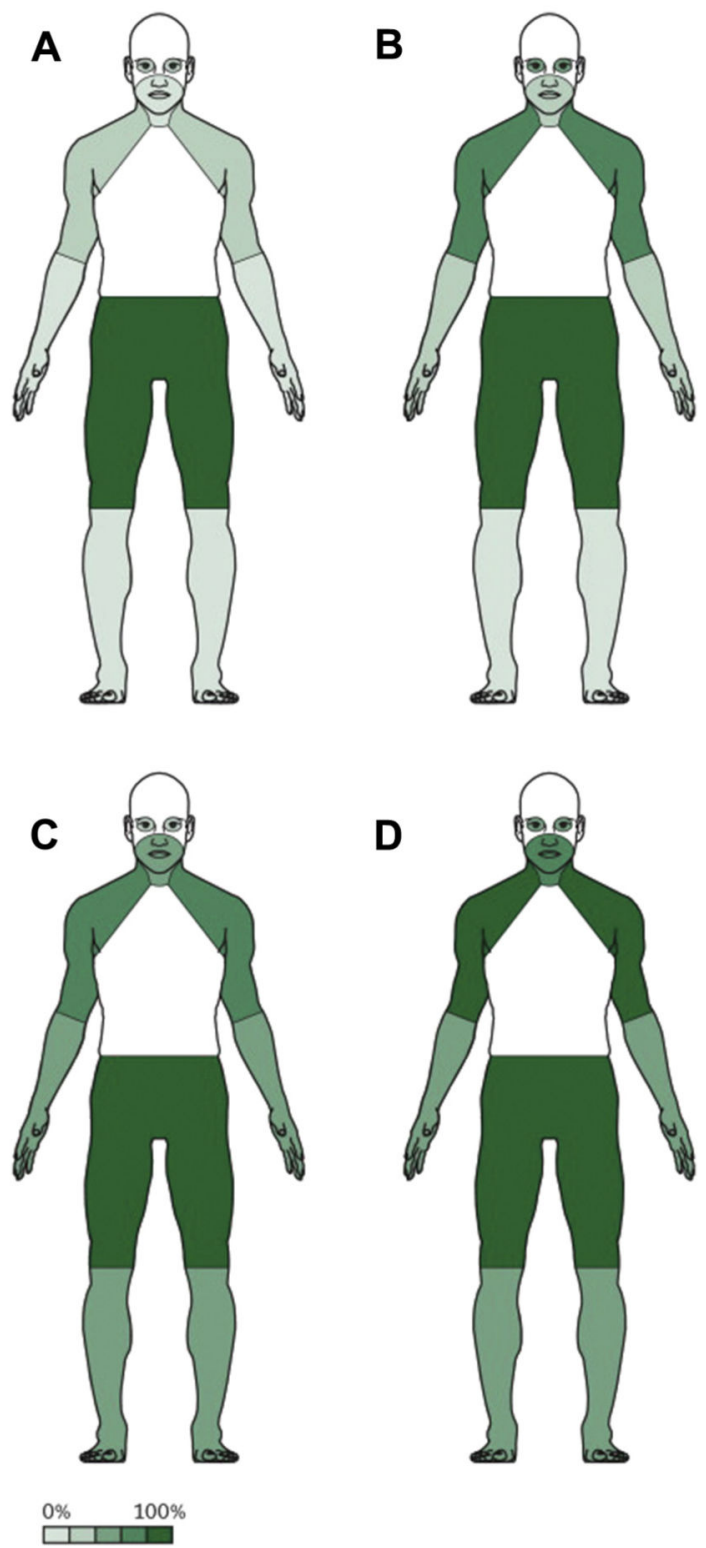

Fig. 1.

Spreading of symptoms in patients with NT-LEMS and SCLC-LEMS. Frequency of symptoms at 3 months $(A)$ and 12 months $(B)$ in patients with NT-LEMS, and frequency of symptoms at 3 months $(C)$ and 12 months $(D)$ in patients with SCLC-LEMS. The percentages describe the approximate proportion of patients who have that symptom within the given timeframe. (From Titulaer MJ, Lang B, Verschuuren JJ. Lambert-Eaton myasthenic syndrome: from clinical characteristics to therapeutic strategies. Lancet Neurol 2011;10(12):1099; Reprinted with permission from Elsevier (The Lancet).) 

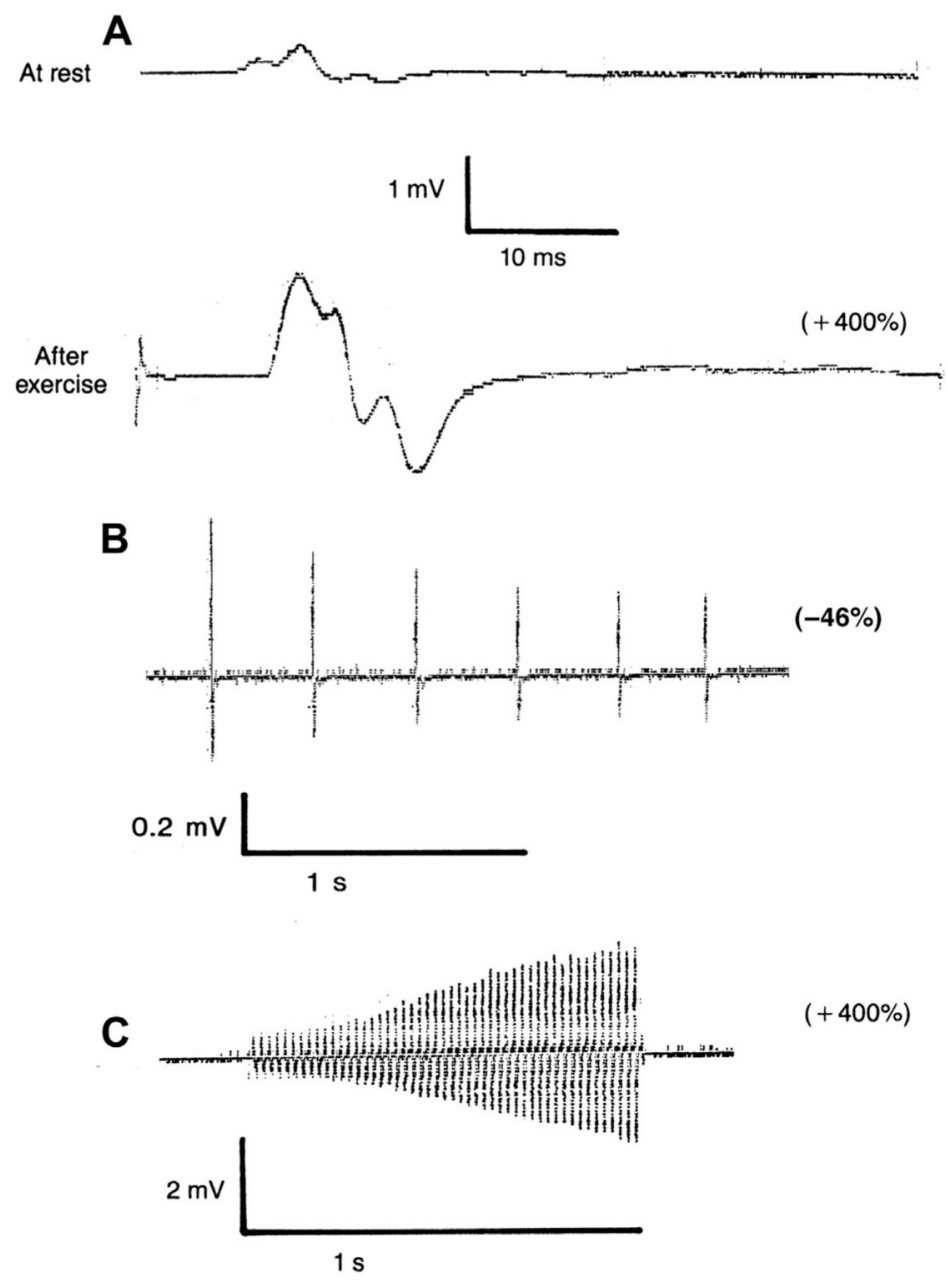

$(+400 \%)$

Fig. 2.

Typical repetitive nerve stimulation pattern in the abductor digiti minimi muscle in LEMS. (A) Low compound action potential amplitude at rest, marked incremental response $(+400$ $\mathrm{mg})$ after 30 seconds of exercise. $(B)$ Decremental response $(-46 \%)$ at low-rate stimulation (3 Hz). (C) Marked incremental response (+400\%) at high-rate stimulation $(50 \mathrm{~Hz})$. (From Oh SJ. Treatment and management of the neuromuscular junction. In: Bertorini TE, editor. Neuromuscular disorders. Treatment and management. Philadelphia: Elsevier-Saunders; 2011. p. 323; with permission.) 


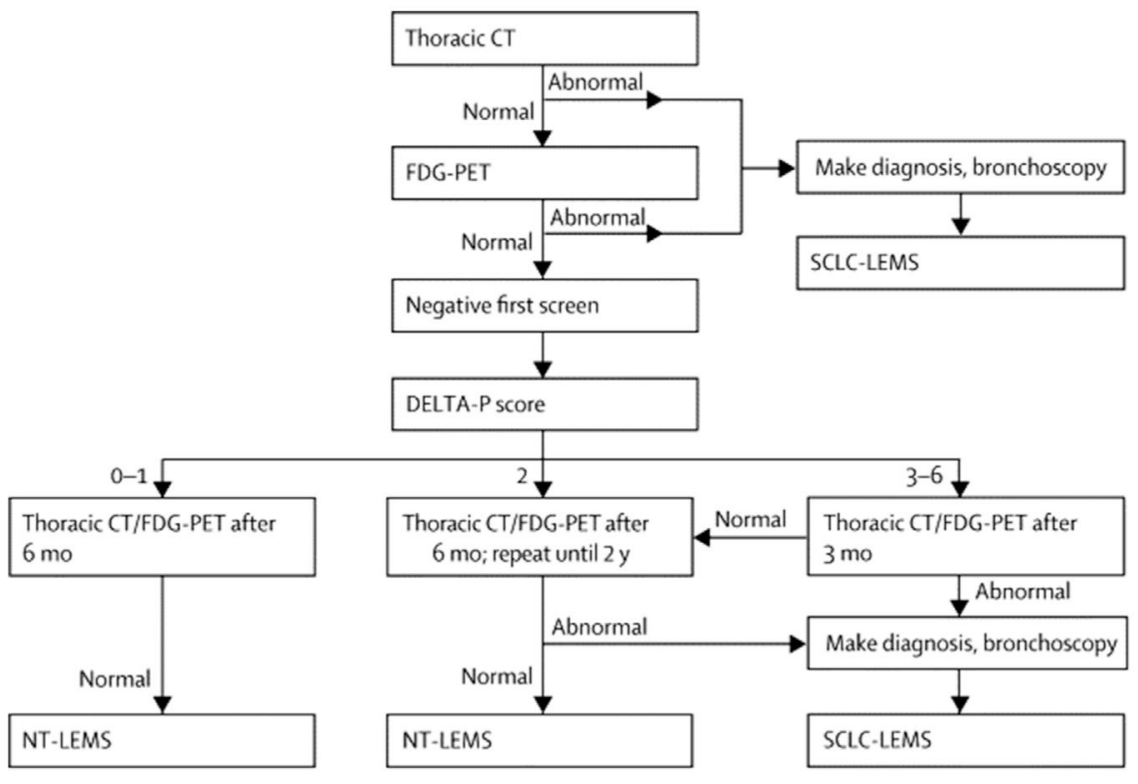

Fig. 3.

Flowchart of recommended screening for SCLC in patients with LEMS. CT, computed tomography; FDG, F-fluorodeoxyglucose. (From Titulaer MJ, Lang B, Verschuuren JJ. Lambert-Eaton myasthenic syndrome: from clinical characteristics to therapeutic strategies. Lancet Neurol 2011;10(12):1103; Reprinted with permission from Elsevier (The Lancet).) 


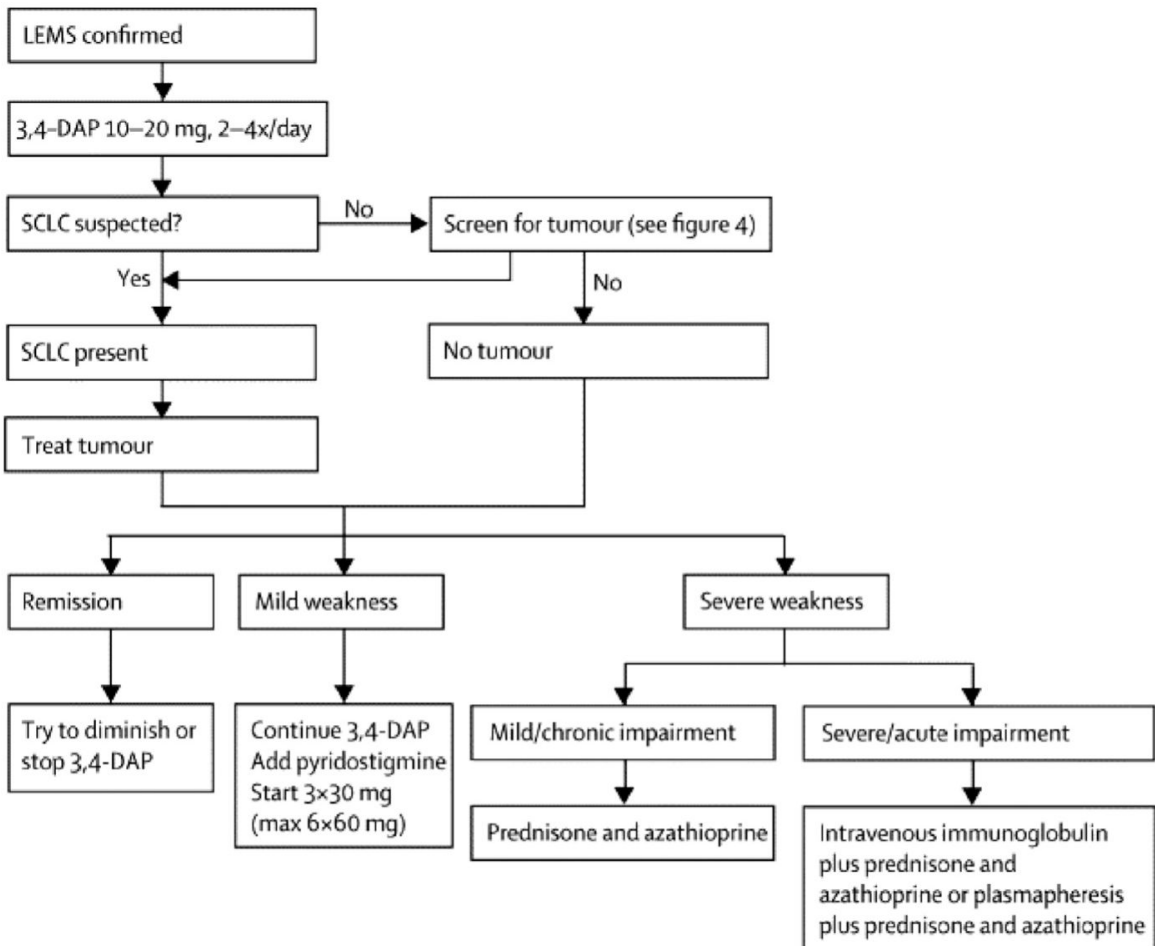

Fig. 4.

Treatment scheme for LEMS. (From Titulaer MJ, Lang B, Verschuuren JJ. Lambert-Eaton myasthenic syndrome: from clinical characteristics to therapeutic strategies. Lancet Neurol 2011;10(12):1104; Reprinted with permission from Elsevier (The Lancet).) 